\title{
Composite materials in flexible multibody systems
}

\author{
Maria Augusta Neto ${ }^{\mathrm{a}, *}$, Jorge A.C. Ambrósio ${ }^{\mathrm{b}}$, Rogério P. Leal ${ }^{\mathrm{a}}$ \\ a Department of Mechanical Engineering, Faculdade de Ciência e Tecnologia da Universidade de Coimbra (Polo II), 3020 Coimbra, Portugal \\ ${ }^{\mathrm{b}}$ Institute of Mechanical Engineering, Instituto Superior Técnico, Av. Rovisco Pais, 1049-001 Lisboa, Portugal
}

\begin{abstract}
In this work the flexible multibody dynamics formulations of complex models are extended to include elastic components made of laminated composite materials. The only limitation for the deformation of a structural member is that it must be elastic and linear when described in a body fixed frame. A finite element model for each flexible body is obtained such that the nodal coordinates are described with respect to the body fixed frame and the inertia terms involved in the mass matrix and gyroscopic force vector use a diagonalized mass description of the inertia terms. The coupling between the flexible body deformation and its rigid body motion is described using only standard finite element parameters obtained with a commercial finite element code. These elements include composite material shells and beams. For composite material beam elements, the properties of their sections are found using an asymptotic procedure proposed by Hodges. The component mode synthesis is used to reduce the number of generalized coordinates to a reasonable dimension for complex shaped structural models of flexible bodies. The kinematic constraints between the different system components are introduced and the equations of motion of the flexible multibody system are solved using an augmented Lagrangean formulation. Finally, the methodology is applied to the analysis of the deployment of a synthetic aperture radar (SAR) Antenna and the results are discussed.
\end{abstract}

(C) 2005 Published by Elsevier B.V.

Keywords: Multibody dynamics; Mode component synthesis; Plate/shell elements; Satellite antenna deployment; Composite material beam element

\section{Introduction}

The use of a multibody methodology to describe the large motion of complex systems that experience structural deformations is advantageous compared to standard non-linear finite element methodologies because it leads to very efficient numerical formulations representing the complete system motion, the relative kinematics between the different components involved, the deformation of the structural members and the inertia coupling. The application of flexible multibody dynamics formulations allows the use of reduction techniques to obtain smaller models while preserving the ability to represent the large rotations of the components with respect to each other or with respect to an inertial reference frame.

Using reference frames fixed to planar flexible bodies, Song and Haug [1] suggested a finite element based methodology yielding coupled gross rigid body motion and small elastic deformations. This idea was further developed by Shabana and Wehage [2] who used the mode component synthesis to reduce the number of generalized coordinates required for the flexible components. Spanos and Tsuha [3] suggested that the selection of modes required for the component mode synthesis implies the solution of an eigenvalue problem. Many authors used this type of formulation to model complex multibody systems [4-8].

Kane et al. [9] showed that although most of the flexible multibody methods can capture the inertia coupling between the elastodynamics of the system components and their large motion, they still produce incorrect results because they

\footnotetext{
${ }^{*}$ Corresponding author.

E-mail addresses: augusta.neto@dem.uc.pt (M.A. Neto), jorge@dem.ist.utl.pt (J.A.C. Ambrósio), rogerio.leal@dem.uc.pt (R.P. Leal).
} 
neglect the dynamic stiffening effects. This comment motivated a very large amount of research work addressing the nature and the solutions of such problems [7,10]. The floating reference frame methods used in flexible multibody dynamics have the ability to lower the geometric non-linearities of the flexible bodies, but they do not eliminate them because the moderate rotation assumption about the floating reference frame is still required [11]. The work of researchers in the finite element community, such as that by Belytschko and Hsieh [12], Simo and Vu-Quoc [13] or Bathe and Bolourchi [14], addressing the same type of problems, can be easily adapted to the framework of flexible multibody dynamics. Recognizing the problem posed and using some of the approaches, well in line with those of the finite element community, Cardona and Geradin proposed formulations for the non-linear flexible bodies using either a geometrically exact model [15] or sub-structuring [16]. Defining it as an absolute nodal coordinate formulation, Shabana [17] used finite rotation nodal coordinates to capture of the geometric non-linear deformations. Another approach taken by Ambrósio and Nikravesh [18] to model geometrically non-linear flexible bodies was to relax the need for the structures to exhibit small moderate rotations about the floating frame by using an incremental finite element approach within the flexible body description.

The structure of the equations of motion for flexible multibody systems obtained with the methods described before for linear elastic deformations with respect to the floating frame, include the mass and stiffness matrices, commonly used for finite element models, the mass and inertia matrices, and the gyroscopic and centrifugal force vectors, always present in multibody formulations, and the inertia coupling terms, which are only encountered in these methods. These coupling terms involve the derivation of matrices, which include the finite element shape functions that are not available in the common finite element literature. As a result, most of the flexible multibody models presented in the literature are made of beam elements only. Using a diagonalized mass formulation for the inertia terms Ambrósio and Gonçalves [19] showed not only that all the terms required in the flexible body equations of motion are readily available from any commercial finite element code but also that any type of linear finite element can be used in the model.

The formulations used for the description of large motion of flexible members have been used in the framework of systems made of standard materials. Recent efforts have been made to describe composite and laminated materials in the framework of multibody systems [20]. The work now presented is a contribution to the representation of flexible multibody systems using composite materials. In particular, it is presented a methodology to describe composite beam elements that follows the work proposed by Cesnik and Hodges [21]. This methodology is applied to demonstrative examples where the flexibility of members of a multibody system plays a decisive role in the behavior of the system.

\section{General equations of motion for flexible multibody systems}

A rigid body is defined by the position of a body fixed reference frame, $\xi \eta \zeta$, and its orientation with relation to an inertia frame, $X Y Z$. The position and orientation of the rigid body $i$ is defined by a set of translation coordinates, represented by $\mathbf{r}_{i}$ and rotational coordinates $\mathbf{p}_{i}$. The body coordinates are then grouped in a vector $\mathbf{q}_{r i}=\left[\mathbf{r}_{i}^{\mathrm{T}} \mathbf{p}_{i}^{\mathrm{T}}\right]^{\mathrm{T}}$. For a flexible body a set of generalized elastic coordinates, designated by $\mathbf{q}_{f}$, has to be used also to fully describe the kinematics of the body. Therefore, a flexible body is described by the coordinates $\mathbf{q}_{i}=\left[\mathbf{q}_{r i}^{\mathrm{T}} \mathbf{q}_{f i}^{\mathrm{T}}\right]^{\mathrm{T}}$. The complete set of coordinates for a multibody system is designated by $\mathbf{q}=\left[\mathbf{q}_{r}^{\mathrm{T}} \mathbf{q}_{f}^{\mathrm{T}}\right]^{\mathrm{T}}$ and includes all the coordinates of the individual bodies of the system.

For a multibody system it is necessary to define a set of kinematic constraints associated to the joints that restrict the relative motion between the bodies of the system [19]

$$
\boldsymbol{\Phi}\left(\mathbf{q}_{r}, \mathbf{q}_{f}, t\right) \equiv \mathbf{0} .
$$

The constraints equations are added to the Lagrange equations using Lagrange multipliers

$$
\mathbf{M} \ddot{\mathbf{q}}+\boldsymbol{\Phi}_{\mathbf{q}}^{\mathrm{T}} \lambda=\mathbf{g}+\mathbf{s}-\underline{\mathbf{K}} \mathbf{q},
$$

where $\underline{\mathbf{K}}$ is the generalized stiffness matrix of the system obtained by the stiffness matrices of the individual bodies $\mathbf{K}_{f f}$, which are multiplied by the generalized elastic coordinates, and null sub-matrices that multiply by the rigid body coordinates in the trailing term of the equation. The second derivatives the constraint equations must also be used in order for the number of equations to be equal to the number of unknowns. These equations are

$$
\ddot{\boldsymbol{\Phi}}\left(\mathbf{q}_{r}, \mathbf{q}_{f}, t\right) \equiv \boldsymbol{\Phi}_{\mathbf{q}} \ddot{\mathbf{q}}-\boldsymbol{\gamma}=\mathbf{0} .
$$

Adding these equations to the system equations of motion, leads to [19]

$$
\left[\begin{array}{ccc}
\mathbf{M}_{r} & \mathbf{M}_{r f} & \boldsymbol{\Phi}_{\mathbf{q}_{r}}^{\mathrm{T}} \\
\mathbf{M}_{f r} & \mathbf{M}_{f f} & \boldsymbol{\Phi}_{\mathbf{q}_{f}}^{\mathrm{T}} \\
\mathbf{\Phi}_{\mathbf{q}_{r}} & \mathbf{\Phi}_{\mathbf{q}_{f}} & \mathbf{0}
\end{array}\right]\left\{\begin{array}{c}
\ddot{\mathbf{q}}_{r} \\
\ddot{\mathbf{u}}^{\prime} \\
\boldsymbol{\lambda}
\end{array}\right\}=\left\{\begin{array}{c}
\mathbf{g}_{r} \\
\mathbf{g}_{f} \\
\gamma
\end{array}\right\}-\left\{\begin{array}{c}
\mathbf{s}_{r} \\
\mathbf{s}_{f} \\
\mathbf{0}
\end{array}\right\}-\left\{\begin{array}{c}
\mathbf{0} \\
\mathbf{K}_{f f} \mathbf{u}^{\prime} \\
\mathbf{0}
\end{array}\right\},
$$


where $\mathbf{u}^{\prime}$ are the nodal displacements of the flexible bodies measured in the respective body fixed coordinate systems. The Jacobian matrix $\boldsymbol{\Phi}_{\mathbf{q}}^{\mathrm{T}}$ and the right-hand-side vector $\gamma$ on Eq. (4) depend on the type of kinematic constraints used. The system equation matrix shows a great number of null elements and zero blocks with fixed size. The Markowitz sparse matrix solver is employed here [22].

The equations of motion for the flexible multibody systems, in Eq. (4) require a large number of coordinates to describe complex models. Using a component mode synthesis method the flexible part of the body is described by a sum of selected modes of vibration as

$$
\mathbf{u}^{\prime}=\mathbf{X w},
$$

where vector $\mathbf{w}$ represents the contributions of the vibration modes towards the nodal displacements and $\mathbf{X}$ is the modal matrix. Due to the reference conditions, the modes of vibration used here are constrained modes, i.e., modes of vibration evaluated using consistent boundary conditions the attachments of the body reference frame to one or more material points in the flexible body. Due to the assumption of linear elastic deformations the modal matrix is invariant. The reduced equations of motion for the flexible body are [19]

$$
\left[\begin{array}{ccc}
\mathbf{M}_{r} & \mathbf{M}_{r f} \mathbf{X} & \mathbf{\Phi}_{\mathbf{q}_{r}}^{\mathrm{T}} \\
\mathbf{X}^{\mathrm{T}} \mathbf{M}_{f r} & \mathbf{I} & \mathbf{X}^{\mathrm{T}} \mathbf{\Phi}_{\mathbf{q}_{f}}^{\mathrm{T}} \\
\boldsymbol{\Phi}_{\mathbf{q}_{r}} & \boldsymbol{\Phi}_{\mathbf{q}_{f}} \mathbf{X} & \mathbf{0}
\end{array}\right]\left\{\begin{array}{c}
\ddot{\mathbf{q}}_{r} \\
\ddot{\mathbf{w}} \\
\boldsymbol{\lambda}
\end{array}\right\}=\left\{\begin{array}{c}
\mathbf{g}_{r} \\
\mathbf{X}^{\mathrm{T}} \mathbf{g}_{f} \\
\boldsymbol{\gamma}
\end{array}\right\}-\left\{\begin{array}{c}
\mathbf{s}_{r} \\
\mathbf{X}^{\mathrm{T}} \mathbf{s}_{f} \\
\mathbf{0}
\end{array}\right\}-\left\{\begin{array}{c}
\mathbf{0} \\
\boldsymbol{\Lambda} \mathbf{w} \\
\mathbf{0}
\end{array}\right\},
$$

where $\boldsymbol{\Lambda}$ is a diagonal matrix with the squares of the natural frequencies associated to the modes of vibration selected. This set of equations for describing the flexible body involves as many generalized coordinates as the number of modes of vibration selected. For a more detailed discussion on the selection of the modes the interested reader is referred to $[8,23,24]$.

\section{Flexible bodies with finite elements in composite materials}

Laminated beams or plate/shells are presently used as structural elements in high performance aerospace, naval and civil applications, where high strength and high stiffness to weight ratios are required [25]. In the approach proposed the flexibility is included in the multibody system assuming the hypothesis of small displacements and rotations with respect to a body attached reference frame. Furthermore, it is assumed here that the composite materials, made of orthotropic lamina, are linear elastic and that delamination does not occur.

\subsection{Orthotropic lamina}

Composite materials consisting of stiff and strong fibers embedded in a compatible matrix are named fibrous composites. Laminated composites are made with layers of different materials and/or different orientations. For the $k$ th layer of orthotropic material shown in Fig. 1, the material presents three orthogonal planes of elastic symmetry and the constitutive equations in the material coordinate system are written as [26]

$$
\left[\begin{array}{l}
\bar{\sigma}_{11} \\
\bar{\sigma}_{22} \\
\bar{\sigma}_{33} \\
\bar{\sigma}_{23} \\
\bar{\sigma}_{13} \\
\bar{\sigma}_{12}
\end{array}\right]_{k}=\left(\left[\begin{array}{cccccc}
\bar{c}_{11} & \bar{c}_{12} & \bar{c}_{13} & 0 & 0 & 0 \\
\bar{c}_{21} & \bar{c}_{22} & \bar{c}_{23} & 0 & 0 & 0 \\
\bar{c}_{31} & \bar{c}_{32} & \bar{c}_{33} & 0 & 0 & 0 \\
0 & 0 & 0 & \bar{c}_{44} & 0 & 0 \\
0 & 0 & 0 & 0 & \bar{c}_{55} & 0 \\
0 & 0 & 0 & 0 & 0 & \bar{c}_{66}
\end{array}\right]\left[\begin{array}{c}
\bar{\varepsilon}_{11} \\
\bar{\varepsilon}_{22} \\
\bar{\varepsilon}_{33} \\
\bar{\varepsilon}_{23} \\
\bar{\varepsilon}_{13} \\
\bar{\varepsilon}_{12}
\end{array}\right]\right)_{k}
$$

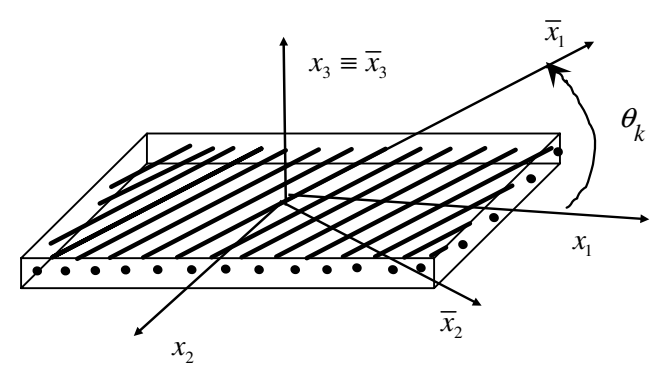

Fig. 1. An orthotropic layer with principal material directions. 
where the indices 1, 2 and 3 are the axis of the system that form the three orthogonal planes of material symmetry. The elastic coefficients $\bar{c}_{i j}$ in the last equation are related to the modulus of elasticity $E_{i}$, Poisson's ratio $v_{i j}$ and shear modulus $G_{i j}$.

The coordinate system used in the solution of the problem does not coincide with the material coordinate system. Furthermore, the composite laminates have several layers; each with different orientation angle $\theta_{k}$ of their material coordinates with respect to the laminate coordinate system. Thus, there is a need to establish transformation relations among stresses and strains in one coordinate system to the corresponding quantities in another coordinate system. Upon transformation, the lamina constitutive equations are expressed in the global coordinate system as

$$
\left[\begin{array}{l}
\sigma_{11} \\
\sigma_{22} \\
\sigma_{33} \\
\sigma_{23} \\
\sigma_{13} \\
\sigma_{12}
\end{array}\right]_{k}=\left(\left[\begin{array}{cccccc}
c_{11} & c_{12} & c_{13} & 0 & 0 & c_{16} \\
c_{21} & c_{22} & c_{23} & 0 & 0 & c_{26} \\
c_{31} & c_{32} & c_{33} & 0 & 0 & c_{36} \\
0 & 0 & 0 & c_{44} & c_{45} & 0 \\
0 & 0 & 0 & c_{54} & c_{55} & 0 \\
c_{61} & c_{62} & c_{63} & 0 & 0 & c_{66}
\end{array}\right]\left[\begin{array}{c}
\varepsilon_{11} \\
\varepsilon_{22} \\
\varepsilon_{33} \\
2 \varepsilon_{23} \\
2 \varepsilon_{13} \\
2 \varepsilon_{12}
\end{array}\right]\right)_{k}
$$

where the $c_{i j}$ are the transformed elastic coefficients referred to the $\left(x_{1}, x_{2}, x_{3}\right)$ coordinate system, which are related to the elastic coefficients in the material coordinates $\bar{c}_{i j}$, by

$$
\mathbf{C}_{k}=\left(\mathbf{T} \overline{\mathbf{C}} \mathbf{T}^{\mathrm{T}}\right)_{k},
$$

where $\mathbf{T}$ is the matrix based on the particular transformation between the several coordinate systems. This matrix it is directly dependent on the layer orientation, $\theta_{k}$, where the index $k$ refers to the $k$ th layer.

\subsection{Platelshell element}

The shell element used in this work is based on Mindlin-Reissner plate theory where only $C^{0}$ continuity is required for the approximation of the kinematic variables. The element, called discrete shear triangle (DST) degenerates automatically [27] to the discrete Kirchhoff triangle (DKT) element if transverse shear effects are not important. The DST element was extended to the treatment of plate and shell structures with the incorporation of membrane stiffness [28]. The first-order theory leads to constant transverse shear stress, which violates equilibrium at the free surfaces of the plate, and continuity requirements of the interlaminar shear stress. To account for the discrepancy between the constant state of shear strains in the first-order theory, and the quadratic or higher-order distribution of shear strains of elasticity theory, shear correction factors are introduced. Significance research has addressed the selection of exact or improved values of these factors for laminated plates [28].

In the Mindlin-Reissner or first-order shear deformation laminated plate theory (FSDT) the assumption that "the normal to the mid-surface of the undeformed plate remains straight but not necessarily normal to the mid-surface of the deformed plate" leads to the following definition of the displacement [29],

$$
\begin{aligned}
& u_{1}^{\prime \prime}\left(x_{\mathrm{m}}^{\prime \prime}, t\right)=u_{1}^{\prime \prime}\left(x_{\alpha}^{\prime \prime}, t\right)+x_{3}^{\prime \prime} \phi_{1}^{\prime \prime}\left(x_{\alpha}^{\prime \prime}, t\right), \\
& u_{2}^{\prime \prime}\left(x_{\mathrm{m}}^{\prime \prime}, t\right)=u_{2}^{\prime \prime 0}\left(x_{\alpha}^{\prime \prime}, t\right)+x_{3}^{\prime \prime} \phi_{2}^{\prime \prime}\left(x_{\alpha}^{\prime \prime}, t\right), \\
& u_{3}^{\prime \prime}\left(x_{\mathrm{m}}^{\prime \prime}, t\right)=u_{3}^{\prime \prime 0}\left(x_{\alpha}^{\prime \prime}, t\right),
\end{aligned}
$$

where the Latin subscripts take values of 1,2 and 3 and Greek subscripts of 1 and 2. The displacement component of a point of the mid-surface in the $x_{i}^{\prime \prime}$ direction is denoted by $u_{i}^{\prime \prime 0}$ and the rotations of the normal about the $x_{1}^{\prime \prime}$ and $x_{2}^{\prime \prime}$ axes are represented by $\phi_{1}^{\prime \prime}$ and $-\phi_{2}^{\prime \prime}$, respectively, as seen in Fig. 2.

The finite element used is triangular and has five degrees of freedom per node, i.e., the nodal displacements $u_{1}^{\prime \prime 0}, u_{2}^{\prime \prime 0}, u_{3}^{\prime \prime}$, $\phi_{1}^{\prime \prime}$ and $\phi_{2}^{\prime \prime}$ defined in the element local axes. When one node is common to several elements with different orientations, the transformation of the displacement to a global system, axes $x_{i}^{\prime}$, must be done before the assembly. It is necessary to introduce a third rotation, $\phi_{3}^{\prime}$ in the global system, to account the possible components projection of the rotations $\phi_{1}^{\prime \prime}, \phi_{2}^{\prime \prime}$ on the global system. In this context the relation between local and global components, at element level, takes the form

$$
\mathbf{u}^{\prime(e)}=\mathbf{R}^{(e)} \mathbf{u}^{(e)}
$$

with

$$
\mathbf{u}^{\prime \prime(e)}=\left[\begin{array}{lllll}
u_{1}^{\prime \prime 0} & u_{2}^{\prime \prime 0} & u_{3}^{\prime \prime 0} & \phi_{1}^{\prime \prime} & \phi_{2}^{\prime \prime}
\end{array}\right]^{(e) \mathrm{T}}, \quad \mathbf{u}^{\prime(e)}=\left[\begin{array}{llllll}
u_{1}^{\prime 0} & u_{2}^{\prime 0} & u_{3}^{\prime 0} & \phi_{1}^{\prime} & \phi_{2}^{\prime} & \phi_{3}^{\prime}
\end{array}\right]^{(e) \mathrm{T}},
$$

where $\mathbf{R}^{(e)}$ is the transformation matrix between the two systems. This relation must be used only for non-coplanar nodes, for instance if the local and global system are the same, the projection of the local rotations $\theta_{1}^{\prime \prime}, \theta_{2}^{\prime \prime}$ over the global axes $x_{3}^{\prime}$ 


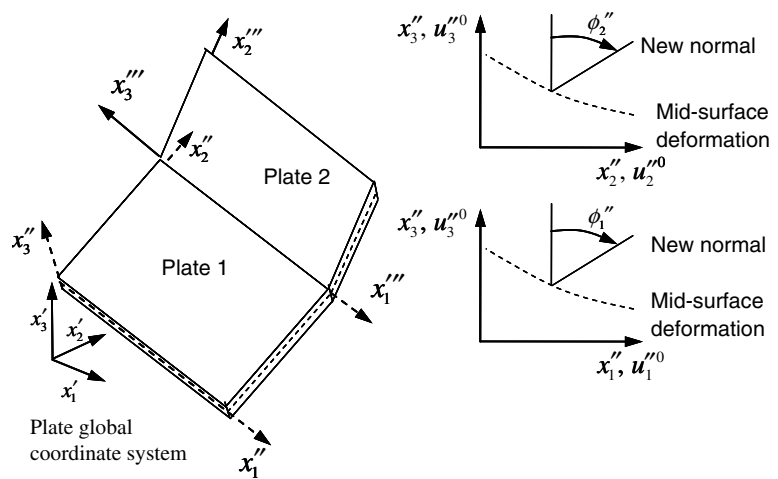

Fig. 2. Global and local axes, local displacements for the Mindlin-Reissner theory.

are zero. Therefore, for coplanar nodes that are part of different elements it is important to introduce a fictitious rotation at the local level to avoid the singularity of the stiffness matrix [30].

Using the Hamilton's principle with appropriate interpolation functions [26] and following standard displacement based finite procedures, it is possible to obtain the global equilibrium system of linear equations in the form

$$
\mathbf{M}_{f f} \ddot{\mathbf{q}}+\mathbf{K}_{f f} \mathbf{q}=\mathbf{F} .
$$

At element level $(e)$, and in local coordinates $(\xi, \eta)$, the stiffness matrix can be written as

$$
\mathbf{K}_{f f}^{(e)}=\int_{0}^{1} \int_{0}^{1-\eta}\left[\begin{array}{ccc}
\mathbf{B}_{\mathrm{m}}^{\mathrm{T}} \mathbf{D}_{\mathrm{m}} \mathbf{B}_{\mathrm{m}} & \mathbf{B}_{\mathrm{m}}^{\mathrm{T}} \mathbf{D}_{\mathrm{mb}} \mathbf{B}_{\mathrm{b}} & \mathbf{0} \\
\mathbf{B}_{\mathrm{b}}^{\mathrm{T}} \mathbf{D}_{\mathrm{bm}} \mathbf{B}_{\mathrm{m}} & \mathbf{B}_{\mathrm{b}}^{\mathrm{T}} \mathbf{D}_{\mathrm{b}} \mathbf{B}_{\mathrm{b}} & \mathbf{0} \\
\mathbf{0} & \mathbf{0} & \mathbf{B}_{\mathrm{s}}^{\mathrm{T}} \mathbf{D}_{\mathrm{s}} \mathbf{B}_{\mathrm{s}}
\end{array}\right]^{(e)}|\mathbf{J}| \mathrm{d} \xi \mathrm{d} \eta,
$$

which is written in the compact form as

$$
\mathbf{K}_{f f}^{(e)}=\int_{0}^{1} \int_{0}^{1-\eta}\left(\mathbf{B}^{\mathrm{T}} \mathbf{D B}\right)^{(e)}|\mathbf{J}| \mathrm{d} \xi \mathrm{d} \eta .
$$

Matrix $\mathbf{B}$ is the strain-displacement matrix, $\mathbf{D}$ the elasticity matrix and $|\mathbf{J}|$ the determinant of the Jacobian matrix, which for triangular elements is twice the area of the element. The subscripts $\mathrm{m}, \mathrm{b}$ and s stand for membrane, bending and shear, respectively, and

$$
\mathbf{B}^{(e)}=\mathbf{L} \mathbf{S}^{(e)},
$$

where $\mathbf{S}^{(e)}$ is the shape function matrix, that depends on the choice of finite element and laminate theory, and $\mathbf{L}$ a matrix of differential operators. The constitutive equation for the laminate at element level is given by [27]

$$
\left[\begin{array}{c}
\mathbf{N} \\
\mathbf{M} \\
\mathbf{Q}
\end{array}\right]^{(e)}=\left[\begin{array}{ccc}
\mathbf{D}_{\mathrm{m}} & \mathbf{D}_{\mathrm{mb}} & 0 \\
\mathbf{D}_{\mathrm{bm}} & \mathbf{D}_{\mathrm{b}} & 0 \\
0 & 0 & \mathbf{D}_{\mathrm{s}}
\end{array}\right]^{(e)}\left[\begin{array}{c}
\boldsymbol{\varepsilon}^{0} \\
\boldsymbol{\varepsilon}^{i} \\
\boldsymbol{\gamma}^{\mathrm{s}}
\end{array}\right]^{(e)}
$$

The sub-matrix $\mathbf{D}_{\mathrm{m}}$ relates resultant membrane forces, $\mathbf{N}$, to membrane strain, $\boldsymbol{\varepsilon}^{0}$, the sub-matrix $\mathbf{D}_{\mathrm{b}}$ relates generalized moments, $\mathbf{M}$, to generalized curvature, $\boldsymbol{\varepsilon}^{i}$, the sub-matrix $\mathbf{D}_{\mathrm{mb}}$ relates resultant membrane forces to generalized curvature, and $\mathbf{D}_{\mathrm{s}}$ relates resultant transverse shear, $\mathbf{Q}$, to shear strains, $\gamma^{\mathrm{s}}$. As each layer may have different properties, the elasticity matrix D must be evaluated by summations carried out through the thickness. Therefore, equivalent single layer theories produce an equivalent stiffness matrix, which is a weighted average of individual layer stiffness through the thickness. All these matrices depend on each layer orientation and are given by

$$
\begin{aligned}
\left(\mathbf{D}_{\mathrm{m}}, \mathbf{D}_{\mathrm{b}}, \mathbf{D}_{\mathrm{mb}}, \mathbf{D}_{\mathrm{s}}\right) & =\sum_{k=1}^{n}\left(\mathbf{D}_{\mathrm{m}}, \mathbf{D}_{\mathrm{b}}, \mathbf{D}_{\mathrm{mb}}, \mathbf{D}_{\mathrm{s}}\right)_{k} \\
& =\sum_{k=1}^{n}\left(\left[\begin{array}{lll}
c_{11} & c_{12} & c_{16} \\
c_{21} & c_{22} & c_{26} \\
c_{61} & c_{62} & c_{66}
\end{array}\right] H_{1},\left[\begin{array}{lll}
c_{11} & c_{12} & c_{16} \\
c_{21} & c_{22} & c_{26} \\
c_{61} & c_{62} & c_{66}
\end{array}\right] H_{2},\left[\begin{array}{lll}
c_{11} & c_{12} & c_{16} \\
c_{21} & c_{22} & c_{26} \\
c_{61} & c_{62} & c_{66}
\end{array}\right] H_{3},\left[\begin{array}{ll}
c_{44} & c_{45} \\
c_{54} & c_{55}
\end{array}\right] H_{1}\right)
\end{aligned}
$$

with

$$
H_{n}=\int_{h_{l-1}}^{h_{l}}\left(x_{3}^{n-1}\right) \mathrm{d} z=\frac{1}{n}\left(h_{l+1}^{n}-h_{l}^{n}\right)
$$




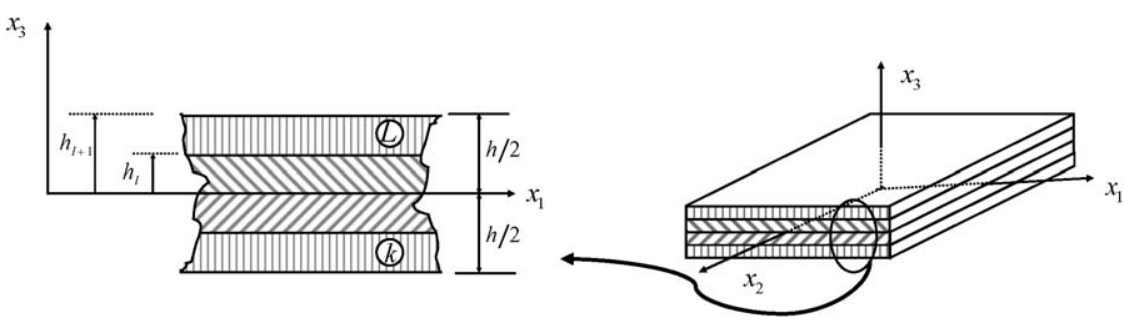

Fig. 3. Coordinate system and layer numbering used for a typical laminated plate.

where $h_{1}$ is defined with the help of Fig. 3. The $x_{3}$-axis is positive upward from the mid-plane and the $L$ th layer is located between the points $x_{3}=h_{1}$ and $x_{3}=h_{1+1}$ in the thickness direction.

At the element level $(e)$, and in local coordinates $(\xi, \eta)$, the consistent mass matrix is

$$
\mathbf{M}_{f f}^{(e)}=\int_{0}^{1} \int_{0}^{1-\eta} \rho^{(e)}\left(\mathbf{S}^{\mathrm{T}} \mathbf{m} \mathbf{S}\right)^{(e)}|\mathbf{J}| \mathrm{d} \xi \mathrm{d} \eta
$$

where $\mathbf{m}$ is the matrix containing the inertial terms and $\rho$ is the specific mass of the element. A diagonalization procedure is used to obtain the lumped mass [31].

\subsection{Beam element}

In the finite element analysis of 1-D beam problems the Timoshenko first-order shear deformation theory (FSDT) is used. Euler-Bernoulli theory ignores transverse shear deformation, which have significant effect on the behavior of the fiber reinforced laminated structures, due to the large difference in the elastic properties between fiber and matrix material. This leads to high ratios of in-plane Young's modulus to transverse modulus for most applications. When these high ratios are coupled with depth effects this theory is inadequate for the analysis of highly anisotropic beams. To improve the situation, the Timoshenko first-order shear deformation theory (FSDT) is applied to multilaminated anisotropic beams. In this theory, the transverse strains are constants through the depth of the beam, hence, the transverse shears are also constant. This discrepancy is overcome by introducing shear correction factors.

Consider a set of unit vectors $\mathbf{e}_{1}, \mathbf{e}_{2}$, and $\mathbf{e}_{3}$ attached to the centroid of the beam cross-section being $\mathbf{e}_{1}$ aligned with the beam axis while $\mathbf{e}_{2}$ and $\mathbf{e}_{3}$ define the plane of the cross-section, as depicted in Fig. 4. Let $u_{1}\left(x_{1}, x_{2}, x_{3}, t\right), u_{2}\left(x_{1}, x_{2}, x_{3}, t\right)$ and $u_{3}\left(x_{1}, x_{2}, x_{3}, t\right)$ be the displacement components of an arbitrary point of the beam in the $\mathbf{e}_{1}, \mathbf{e}_{2}$, and $\mathbf{e}_{3}$ directions, respectively. The displacement field in the plane of the cross-section is described by

$$
\begin{aligned}
& u_{1}\left(x_{\mathrm{m}}, t\right)=u_{1}^{0}\left(x_{1}, t\right)+x_{3} \phi_{2}\left(x_{1}, t\right)-x_{2} \phi_{3}\left(x_{1}, t\right), \\
& u_{2}\left(x_{\mathrm{m}}, t\right)=u_{2}^{0}\left(x_{1}, t\right)-x_{3} \phi_{1}\left(x_{1}, t\right), \\
& u_{3}\left(x_{\mathrm{m}}, t\right)=u_{3}^{0}\left(x_{1}, t\right)+x_{2} \phi_{1}\left(x_{1}, t\right),
\end{aligned}
$$

where $u_{i}^{0}$ is the displacement of the centroid in the direction $x_{i}, \phi_{1}$ is the angle of twist of the cross-section about $x_{1}$. Rotations of the cross-sections, $\phi_{2}\left(x_{1}, t\right)$ and $\phi_{3}\left(x_{1}, t\right)$, are positive about the axes $\mathbf{e}_{2}$ and $\mathbf{e}_{3}$, respectively.

Using Hamilton's principle with appropriate interpolation functions [27] and following the standard displacement finite procedures, it is possible to obtain the equilibrium system of linear equations in the form of Eq. (14). According to Eq. (16), at element level $(e)$, and in local coordinates $(\xi)$, the stiffness matrix is written as

$$
\mathbf{K}_{f f}^{(e)}=\int_{-1}^{1}\left(\mathbf{B}^{\mathrm{T}} \mathbf{D B}\right)^{(e)}|\mathbf{J}| \mathrm{d} \xi
$$

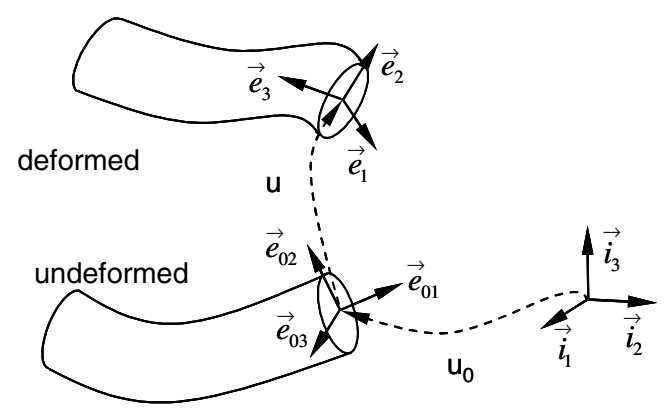

Fig. 4. Beam local coordinate frames and point displacements. 
The elasticity matrix $\mathbf{D}$ is computed using the code VABS developed by $\mathrm{Yu}$ and Hodges [32]. This is a 2-D finite element code that computes the stiffness tensor $\mathbf{D}$ for arbitrary materials and geometries [32]. According to this author, in Timoshenko theory, matrix $\mathbf{D}$ can be expressed as

$$
\mathbf{D}=\left[\begin{array}{cc}
\mathbf{X} & \mathbf{F} \\
\mathbf{F}^{\mathrm{T}} & \mathbf{G}
\end{array}\right]
$$

where the sub-matrix $\mathbf{X}$ relates the resultant axial force, $\mathbf{N}_{x x}$, with the axial strain and the generalized curvatures, and the generalized moments, $\mathbf{M}=\left[\begin{array}{lll}M_{x x} & M_{y y} & M_{z z}\end{array}\right]^{\mathrm{T}}$ to the axial strain and curvatures. The sub-matrix $\mathbf{F}$ relates the resultant axial force and the generalized moments with the transverse shear, and the sub-matrix $\mathbf{G}$ relates the resultant transverse shear force to the shear strains, $\gamma=\left[\begin{array}{lll}2 \varepsilon_{12} & 2 \varepsilon_{13}\end{array}\right]^{\mathrm{T}}$. These matrices are computed using the 2-D Finite Element code VABS over the section of the beam. In the analysis of the beam cross-section, the classical, $w_{0}$, and first-order warping, $w_{1}$, fields are discretized as

$$
\begin{aligned}
& w_{0}\left(x_{1}, x_{2}, x_{3}\right)=\mathbf{N}\left(x_{2}, x_{3}\right) \mathbf{V}_{0}\left(x_{1}\right), \\
& w_{1}\left(x_{1}, x_{2}, x_{3}\right)=\mathbf{N}\left(x_{2}, x_{3}\right) \mathbf{V}_{1}\left(x_{1}\right)
\end{aligned}
$$

with $\mathbf{N}$ representing the element shape functions and $\mathbf{V}_{0}$ and $\mathbf{V}_{1}$ as column matrices of the nodal values of the warping displacements over the cross-section. After the substitution in the strain energy of the cross-section the warping field and the minimization of this energy with respect to the warping field and after using the variational-asymptotic method of Berdichevsky [33] the warping functions can be determined asymptotically in terms of 1-D strain measures as

$$
\begin{aligned}
& \mathbf{V}_{0}=\hat{\mathbf{V}}_{0} \boldsymbol{\varepsilon}, \\
& \mathbf{E} \hat{\mathbf{V}}_{0}=\left(\mathbf{H} \boldsymbol{\psi}_{c l} \boldsymbol{\psi}_{c l}^{\mathrm{T}}-\mathbf{I}\right) \mathbf{C}_{h \varepsilon} .
\end{aligned}
$$

After the classical warping calculation, the first-order warping functions can be computed using an identical procedure, as

$$
\begin{aligned}
& \mathbf{V}_{1}=\hat{\mathbf{V}}_{1} \boldsymbol{\varepsilon}^{\prime}, \\
& \mathbf{E} \hat{\mathbf{V}}_{1}=\left(\mathbf{I}-\mathbf{H} \boldsymbol{\psi}_{c l} \boldsymbol{\psi}_{c l}^{\mathrm{T}}\right)\left[\mathbf{C}_{\varepsilon l}^{\mathrm{T}}+\left(\mathbf{C}_{h l}^{\mathrm{T}}-\mathbf{C}_{h l}\right) \hat{\mathbf{V}}_{0}\right]
\end{aligned}
$$

the matrix $\mathbf{E}, \mathbf{C}_{h E}, \mathbf{C}_{h l}, \mathbf{C}_{l l}$ and $\mathbf{C}_{E l}$ contain information about the material properties as well as the geometry of a given cross-section. After the computation of the warping functions and their substitution in the strain energy, matrices $\mathbf{X}, \mathbf{F}$ and $\mathbf{G}$ can be computed as

$$
\mathbf{G}=\mathbf{A}^{*} \mathbf{R}^{-1} \mathbf{A}^{* T},
$$

where the matrix $\mathbf{A}$ and $\mathbf{R}$ are given by

$$
\begin{aligned}
& \mathbf{A}=\mathbf{C}_{\varepsilon \varepsilon}+2 \hat{\mathbf{V}}_{0}^{\mathrm{T}} \mathbf{C}_{h \varepsilon} ; \quad \mathbf{A}^{*}=-\left[\begin{array}{cc}
0 & 0 \\
0 & 0 \\
0 & -1 \\
1 & 0
\end{array}\right] \mathbf{A}, \\
& \mathbf{R}=\hat{\mathbf{V}}_{0}^{\mathrm{T}} \mathbf{C}_{l l} \hat{\mathbf{V}}_{0}+2 \hat{\mathbf{V}}_{1}^{\mathrm{T}} \mathbf{C}_{h l} \hat{\mathbf{V}}_{0},
\end{aligned}
$$

the matrix $\mathbf{F}$ is done by

$$
\mathbf{F}=-\mathbf{B}^{\mathrm{T}} \mathbf{A}^{*-1} \mathbf{G} ; \quad \mathbf{B}=\left(\hat{\mathbf{V}}_{0}^{\mathrm{T}} \mathbf{C}_{h l}+\mathbf{C}_{\varepsilon l}\right) \hat{\mathbf{V}}_{0}
$$

and the matrix $\mathbf{X}$ is given by

$$
\mathbf{X}=\mathbf{A}+\mathbf{F G}^{-1} \mathbf{F}^{\mathrm{T}} .
$$

The interested reader is referred to the details on this procedure that can be found in the reference by Popescu and Hodges [33].

\section{Application to the deployment of a satellite antenna}

The methodology proposed in this work is demonstrated by application to the simulation of the unfolding of a satellite antenna. The synthetic aperture radar (SAR) antenna, presented in Fig. 5, was manufactured by Dornier System Gmbh and it is part of the European research satellite ERS-1. The data for this antenna is reported in the work of Anantharaman and Hiller [4,5] and it has been used in different analyses to demonstrate several multibody formulations features [34]. 


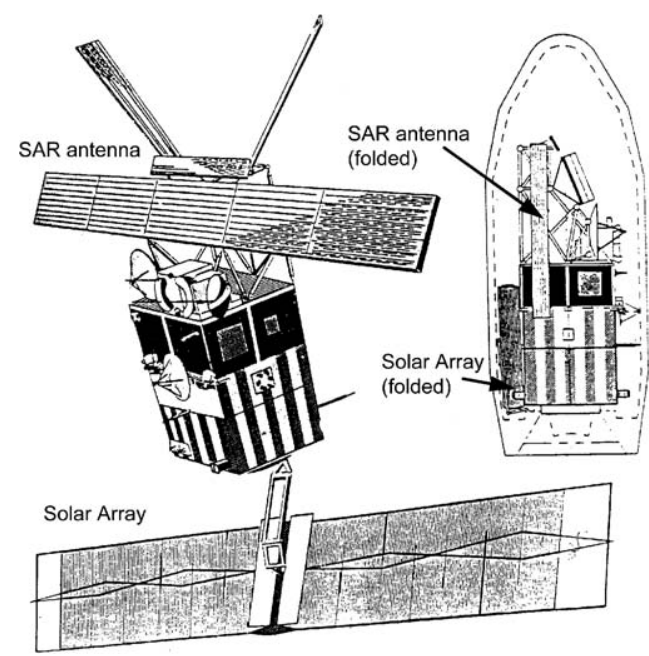

Fig. 5. The European research satellite shown for the folded and unfolded configurations of the SAR antenna.

Both the solar array and the SAR antenna of the ERS-1 satellite have the same configuration and share the same kinematic features. During transportation, the antenna and a solar array are folded, as shown in Fig. 6(b), in order to occupy as little space as possible. After unfolding the mechanical components take the configuration represented in Fig. 6(a). The SAR antenna consists in two identical subsystems, each with three coupled planar four-bar links that unfold two panels on each side. The central panel is attached to the main body of the satellite. Each unfolding system has two degree of freedom, driven individually by actuators located at joints A and B, represented in Fig. 6(a).

The unfolding process consists in two phases. In the first phase the panel 3 is rolled out, around an axis normal to the main body, by a rotational spring-damper-actuator in joint $\mathrm{A}$, while the panel 2 is held down by blocking the joints $\mathrm{D}$ and E. The second phase begins with the joint A blocked, being the panels 2 and 3 swung out to the final position of the first unfolding phase by a rotational spring-damped-actuator.

\subsection{Multibody model of the SAR antenna}

The model used for one half of the folding antenna, schematically depicted Fig. 7, is composed by 12 bodies, denoted $B_{1}, \ldots, B_{12}, 16$ spherical joints, denoted $S_{1}, \ldots, S_{16}$ and 3 revolute joints, referred to as $R_{1}, \ldots, R_{3}$. The central panel is attached to the satellite, defined as body $\mathrm{B}_{1}$, which has much higher mass and inertias. In the first phase of the unfolding
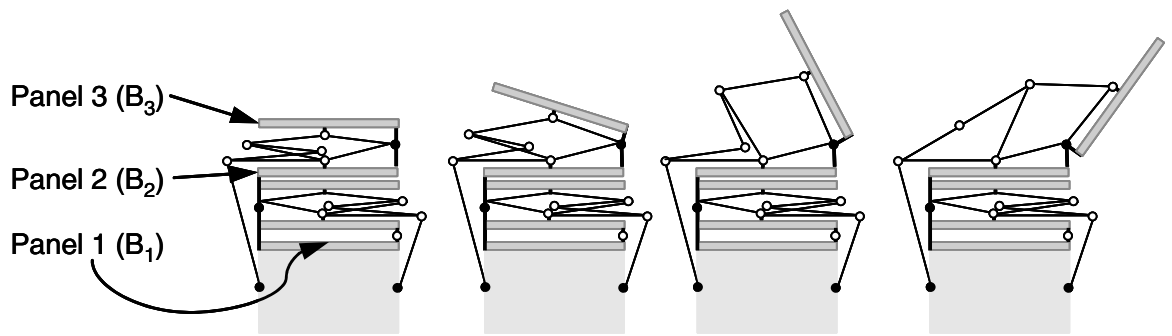

(a)

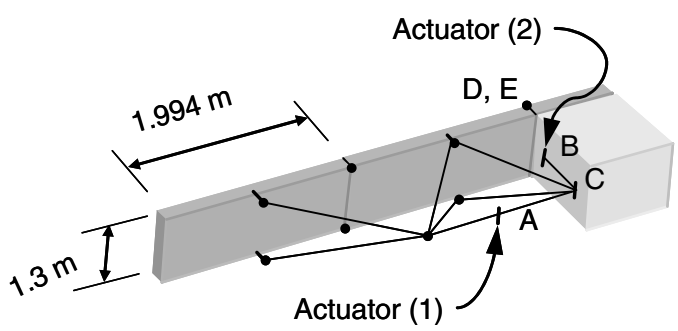

(b)

Fig. 6. The SAR antenna: (a) folded antenna and first part of the unfolding process; (b) half unfolded antenna. 


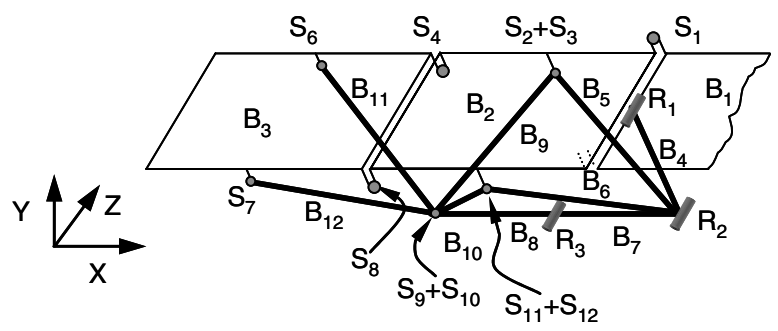

Fig. 7. Multibody model of the SAR antenna.

antenna the rotational spring-damped-actuator acts at revolute joint $\mathrm{R}_{3}$. For the second phase of the unfolding process, revolute joint $R_{3}$ is blocked and the system is moved to the next equilibrium position by a spring-actuator-damped positioned at joint $\mathrm{R}_{1}$.

The basic geometric characteristics of the components of the satellite antenna are presented in Ref. [4] and summarized here as follows. All bodies are made of a carbon fiber reinforced plastic with a specific mass of $1610 \mathrm{~kg} / \mathrm{m}^{3}$. Each panel is $2 \mathrm{~m}$ long by $1.3 \mathrm{~m}$ wide and has a thickness of $2 \mathrm{~mm}$. All spherical joints between the panels, $\mathrm{S}_{1}, \mathrm{~S}_{4}$, $\mathrm{S}_{8}$, etc., are located at the edge of the panels and $0.0705 \mathrm{~m}$ above or below their mid-planes. The spherical joints are supported in place by beams with $6 \mathrm{~mm}$ diameter. The spherical joints between the panels and the trusses, $\mathrm{S}_{2}, \mathrm{~S}_{3}$, $\mathrm{S}_{6}$, etc., are located also at the edge of the panels and $0.025 \mathrm{~m}$ below their mid-plane. The length of the trusses represented by bodies 4 through 12 are $L_{4}=0.273 \mathrm{~m}, L_{5}=L_{6}=L_{9}=L_{10}=L_{11}=L_{12}=1.240 \mathrm{~m}$ and $L_{7}=L_{8}=0.997 \mathrm{~m}$. All truss members have a uniform circular cross-section with the dimensions of $\phi_{4}=20 \mathrm{~mm}, \phi_{7}=\phi_{8}=7 \mathrm{~mm}$ and $\phi_{5}=\phi_{6}=\phi_{9}=\phi_{10}=\phi_{11}=\phi_{12}=6 \mathrm{~mm}$.

In order to fully evaluate the features of the satellite antenna and to compare the results with those obtained in literature, three models are considered. The first model includes all the mechanical components of the antenna represented by rigid bodies. In the second model all components of the satellite are considered flexible, made of isotropic materials. In the last model all members are made of composite materials.

\subsection{Isotropic material model}

In the first flexible multibody model all bodies, except body $\mathrm{B}_{1}$, are assumed to be flexible, made of isotropic linear elastic material with the properties $E=159 \mathrm{GPa}, v=0.38$ and $\rho=1610 \mathrm{~kg} / \mathrm{m}^{3}$. The material properties of the isotropic material are suggested by Anantharaman and Hiller $[4,5]$ as replacement of the panels composite material properties so that the first six natural frequencies and corresponding vibration modes of the model are close to the corresponding natural frequencies and modes of the real panels. The truss members are modeled with two spatial beam elements and two bending modes of vibrations are used for the coordinate reduction. The finite element model of each panel consists of 86 triangular flat shell elements and the 14 modes of vibration associated to the lower natural frequencies of the panels are used to represent panels denoted by $\mathrm{B}_{2}$ and $\mathrm{B}_{3}$.

In the isotropic material model, the actuator acts at revolute joint $\mathrm{R}_{3}$, between bodies $\mathrm{B}_{7}$ and $\mathrm{B}_{8}$, and is represented by a non-linear spring and damper. The non-linear characteristics of the actuator spring are given by a piecewise-linear spring given as

$$
M(\theta, \dot{\theta})=c \dot{\theta}+\left\{\begin{array}{cc}
-2.0 & \theta<-0.2 \\
10.0 \theta & -0.2 \leqslant \theta \leqslant+0.2 \\
2.0 & \theta>0.2
\end{array}\right.
$$

where $M$ is the spring torque, in $\mathrm{N} \mathrm{m}, \theta$ is the actuator angle, in radians, and the damping coefficient is $c=0.5 \mathrm{~N} \mathrm{~m} \mathrm{~s}$.

\subsection{Composite material models}

The dynamic response of the composite material flexible model is dependent on the composite stiffness of the panels which, in turn, is dependent of the layer orientation of the laminate and of the composite material. Although Anantharaman uses an isotropic material to represent the composite material in the satellite antenna model it is indicated in Ref. [4] that the SAR antenna is constructed with a carbon fiber reinforced plastic. The material used in the multibody model is a carbon reinforced plastic IM6/SC1081, the matrix is made of Epoxy SC1081 and the fibers of Carbon IM6. Note that the material model used is not necessarily that of the real satellite antenna, as the characteristics of such material are not publicly available. The properties of the composite material, for a single layer with an orientation of 0 rad relatively to the $X$-axis are: $E_{1}=177 \mathrm{GPa} ; E_{2}=10.8 \mathrm{GPa} ; G_{12}=G_{13}=7.6 \mathrm{GPa} ; G_{23}=8.504 \mathrm{GPa} ; v_{12}=0.27 ; \rho=1600 \mathrm{~kg} / \mathrm{m}^{3}$. Two different laminates with four layers in each, described in Table 1, are considered. 
Table 1

Characteristics of the two lay-ups considered in the applications of the composite panels

\begin{tabular}{|c|c|c|c|c|}
\hline & First layer & Second layer & Third layer & Fourth layer \\
\hline Lay-up 1 & $0^{\circ}$ & $0^{\circ}$ & $0^{\circ}$ & $0^{\circ}$ \\
\hline Lay-up 2 & $0^{\circ}$ & $90^{\circ}$ & $90^{\circ}$ & $0^{\circ}$ \\
\hline Thickness (m) & 0.0005 & 0.0005 & 0.0005 & 0.0005 \\
\hline
\end{tabular}

The actuator for the composite material flexible multibody models that act at revolute joint $\mathrm{R}_{3}$ is also modeled as nonlinear spring and damper actuator, described by piecewise-linear characteristics given by

$$
M(\theta, \dot{\theta})=c \dot{\theta}+ \begin{cases}0.10+9.00(3.12-\theta) & 3.08<\theta \leqslant 3.12, \\ 0.45+60.41(3.08-\theta) & 3.02<\theta \leqslant 3.08, \\ 4.03-5.19(3.02-\theta) & 2.63<\theta \leqslant 3.02, \\ 2.00 & 0.20<\theta \leqslant 2.63, \\ 10.00 \theta & -0.20 \leqslant \theta \leqslant 0.20, \\ -2.00 & -0.20>\theta,\end{cases}
$$

where the damping coefficient is $c=0.5 \mathrm{~N} \mathrm{~m} \mathrm{~s}$. The reason for using an actuator law for the flexible multibody model made of composite material relative to the one used for the isotropic model is discussed later in this work.

\subsection{Dynamic analysis of the rigid and flexible antenna models}

A dynamic analysis of the first stage of the unfolding process, i.e., the deployment of the two first panels due to the action of actuator 1, is performed for the models of rigid and isotropic elastic bodies to allow for the verification of the results obtained against those reported by Anantharaman [4]. Fig. 8 shows the variation of the actuator angle with the simulation time for the flexible and rigid models.

The first observation of the results shows that the models presented in this work lead to a response similar to that obtained by Anantharaman [4]. The small differences between the results of the flexible models with isotropic materials are attributed to the difference between the actuation time laws and finite element meshes used for the panel models. Note that in the original simulation by Anantharaman only 4 QUAD4 rectangular bending elements were used in NASTRAN [35] to obtain 6 modes of vibration whereas in the current investigation, 86 triangular plate/shell elements are used to obtain 14 modes of vibration.

The dynamic responses obtained for the rigid and flexible models are quite different. The unfolding process leads to the equilibrium position corresponding to the alignment between the trusses represented by bodies $\mathrm{B}_{7}$ and $\mathrm{B}_{8}$, which is achieved after $5.5 \mathrm{~s}$, after which bodies $\mathrm{B}_{7}$ and $\mathrm{B}_{8}$ oscillate about such alignment position. During these oscillations panel 3 , represented by body $\mathrm{B}_{3}$, features sudden changes of its angular velocity, as shown in Fig. 9. The abrupt changes in the rotation direction lead to very high constraint reaction forces. The rigid model does not pose any limit to the reaction

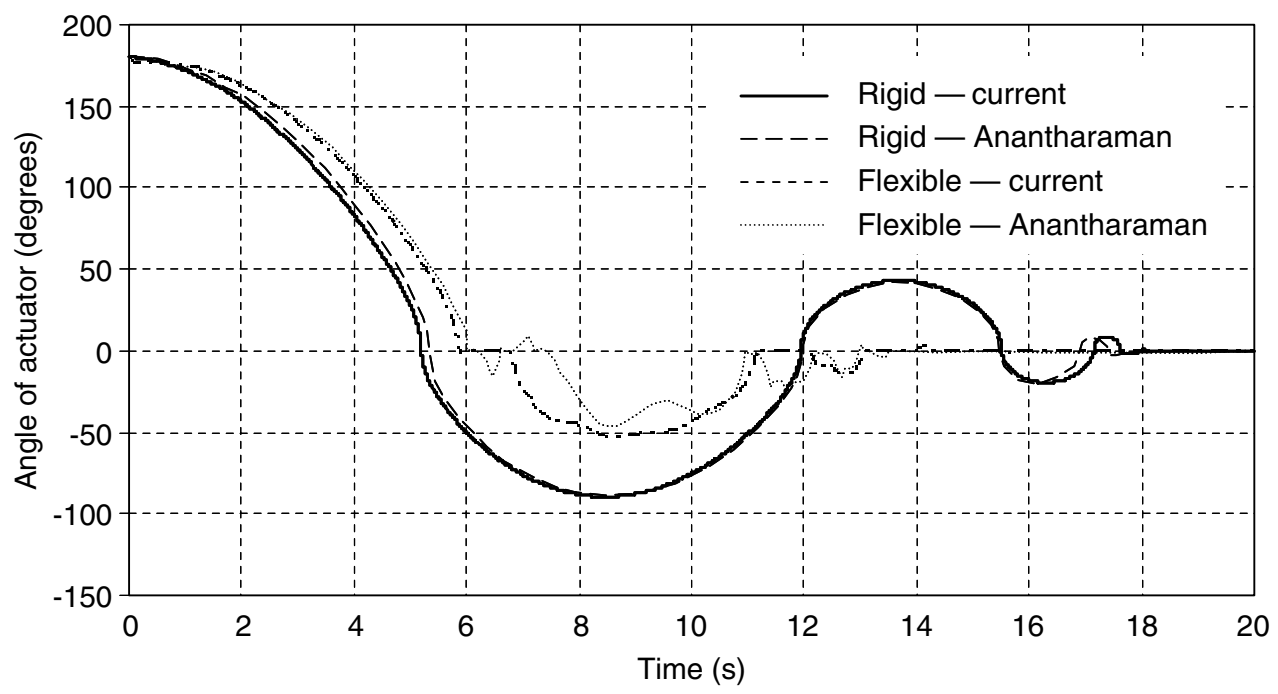

Fig. 8. Angle of the actuator for the rigid and flexible antenna for the first phase of the unfolding process. 


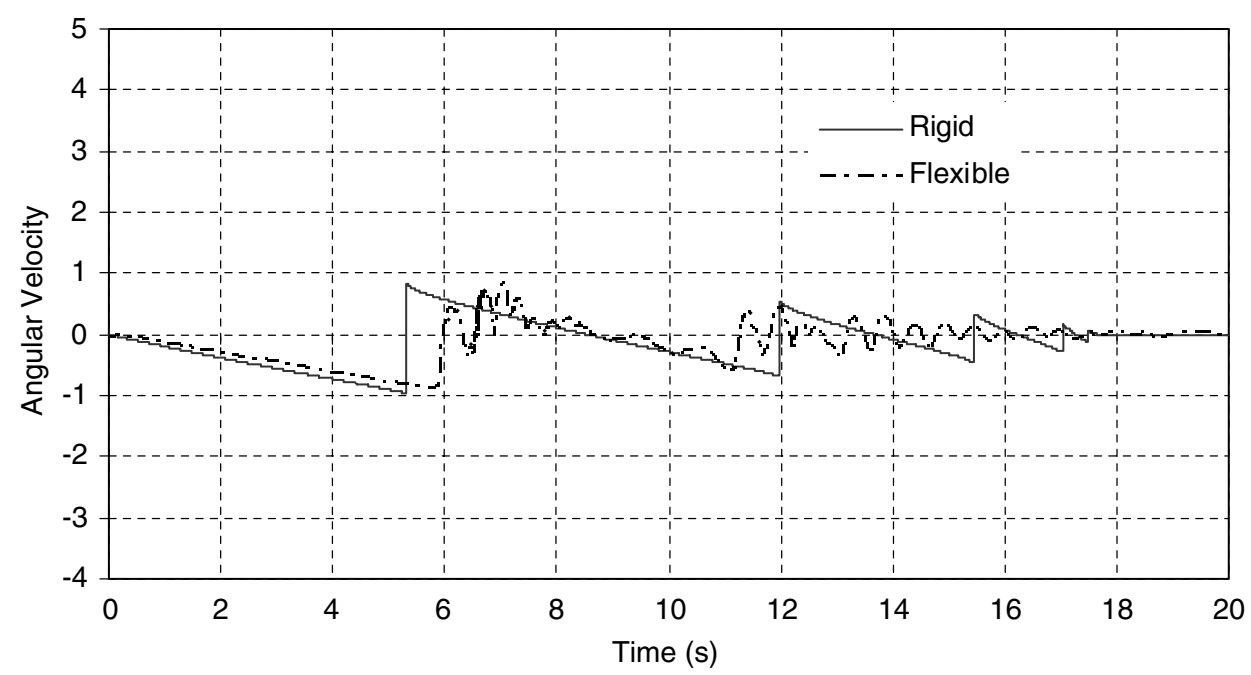

Fig. 9. Angular velocity $\omega_{z}$ of panel 3 for the rigid and flexible antenna during the first deployment phase.

forces and therefore the motion can proceed with very high accelerations. In the elastic model, these forces lead to deformations in the trusses and panels that not only prevent the jumps in angular velocity to take place, but also lead to a transference of kinetic energy from rigid-body motion to elastic vibrations.

In Fig. 10 presents the configurations of the flexible SAR antenna for three different instants of the simulation. There it is clear that when the truss members reach the equilibrium position, at about $5.72 \mathrm{~s}$, the panels start to vibrate about that position, as shown for the instants $6.08 \mathrm{~s}$ and $6.48 \mathrm{~s}$. In this period, the trusses where the actuator spring acts, move as a single flexible body that actually forms a frame structure with the trusses represented by bodies $\mathrm{B}_{5}, \mathrm{~B}_{6}, \mathrm{~B}_{9}$ and $\mathrm{B}_{10}$.

The actuation laws used for the spring-actuators for the rigid and flexible models are different. When the actuation law used for the rigid model was used for the flexible models, the satellite antenna was driven to a different equilibrium state than that obtained in the rigid model. If the limit torque was increased from 2.0 to $4.0 \mathrm{Nm}$ the trusses connected to actuator quickly reached their equilibrium, but the panel 3 hardly moved because the unfolding trusses broke through the panel, as seen in Fig. 11. This is clearly unfeasible because contact would take place between trusses and panels. However, the reported results show that, due to the deformations of the trusses, the undesirable contacts between trusses and panels were possible if the high torques associated to the original actuator have been maintained.

The problems associated to the unfolding of the flexible models have been detected by Anantharaman and Hiller $[4,5]$ and a solution similar to the one adopted here was used in their research. When using composite material models, the problem of the first phase of the unfolding is magnified because not only bending of the panels is of importance but also torsional modes come into play. Fig. 12 presents the variation of the actuator angle during the simulation period for the isotropic and composite models, being the last one represented by the lay-up 2 described in Table 1.

The two simulation results have a similar behavior. However, the simulation results obtained for the flexible model with the composite materials that have the lay-up 2 needs more time to achieve the equilibrium position. This behavior is mainly due to the use of different rotational-spring-actuator models. As mentioned, in the beginning of the motion the flexible model made of composite materials needs a softer rotational actuator to prevent the unfolding trusses from breaking

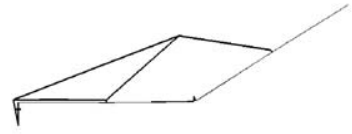

$5.72 \mathrm{~s}$

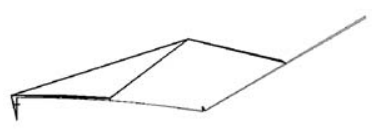

$6.08 \mathrm{~s}$

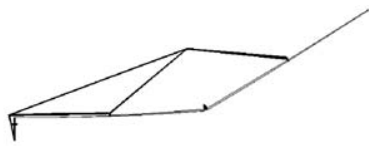

6.46

Fig. 10. Configuration of the isotropic panels with the original damped spring-actuator.
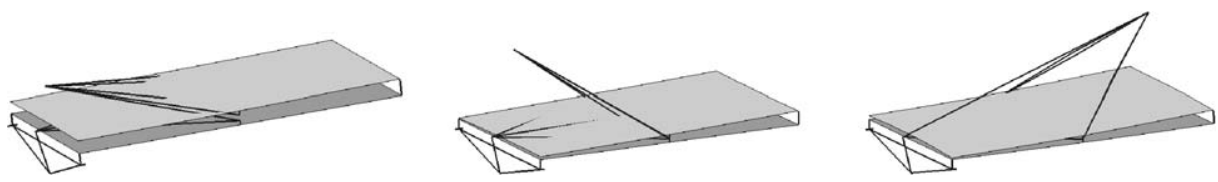

Fig. 11. Configuration of the composite panels with the original damped spring-actuator. 


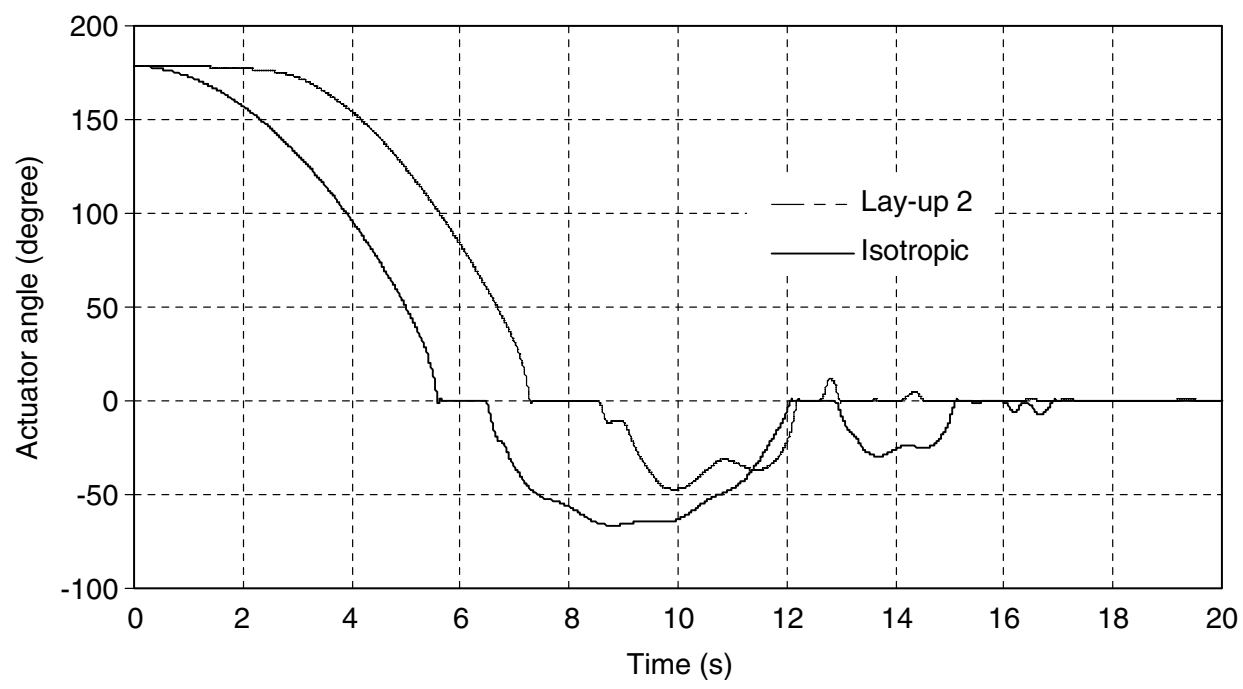

Fig. 12. Actuator angle for the flexible antenna in the first phase with isotropic and composite materials.

through the panel. In fact, the post-processing of simulations results show that, in the composite models, the torsion of the panels is very significant, in contrast to the almost null torsion of the isotropic model. This torsion deformation is also sensitive to the layer orientations, which leads to the different actuator angles history presented in Fig. 13 for composite materials with lay-ups 1 and 2.

It is observed that after the equilibrium positions are reached for both models with composite materials in the period from 7 to $8 \mathrm{~s}$, the direction of rotation of the truss members of the panels made with the lay-up 1 is opposite to that of the model made with the composite material made with lay-up 2. This discrepancy can result from the difference between the vibration modes of the both models. In fact, lay-up 1 has no layers with the $90^{\circ}$ orientation, thus the stiffness of this model in the $Z$-direction is smaller than that observed for lay-up 2. Fig. 14 shows the first phase of the unfolding process for the rigid and for the flexible models, being the deformation of the panels for the flexible model clearly visible.

The second part of the unfolding of the two panels is represented in Fig. 15. Although not fully discussed in this work, the unfolding of the second part of the satellite antenna is similar for the rigid and for the different flexible models; therefore it does not add any further insight in the use of the proposed methodology or in the simulation process. The main reason is that the quick variations of the angular velocities of the panels, observed in Fig. 9, are not present in this phase of the unfolding process. The second part of the unfolding process leads to the alignment of the three panels included in the model. The unfolding of panels 4 and 5 are similar to the process presented before and, therefore, it is not studied here either.

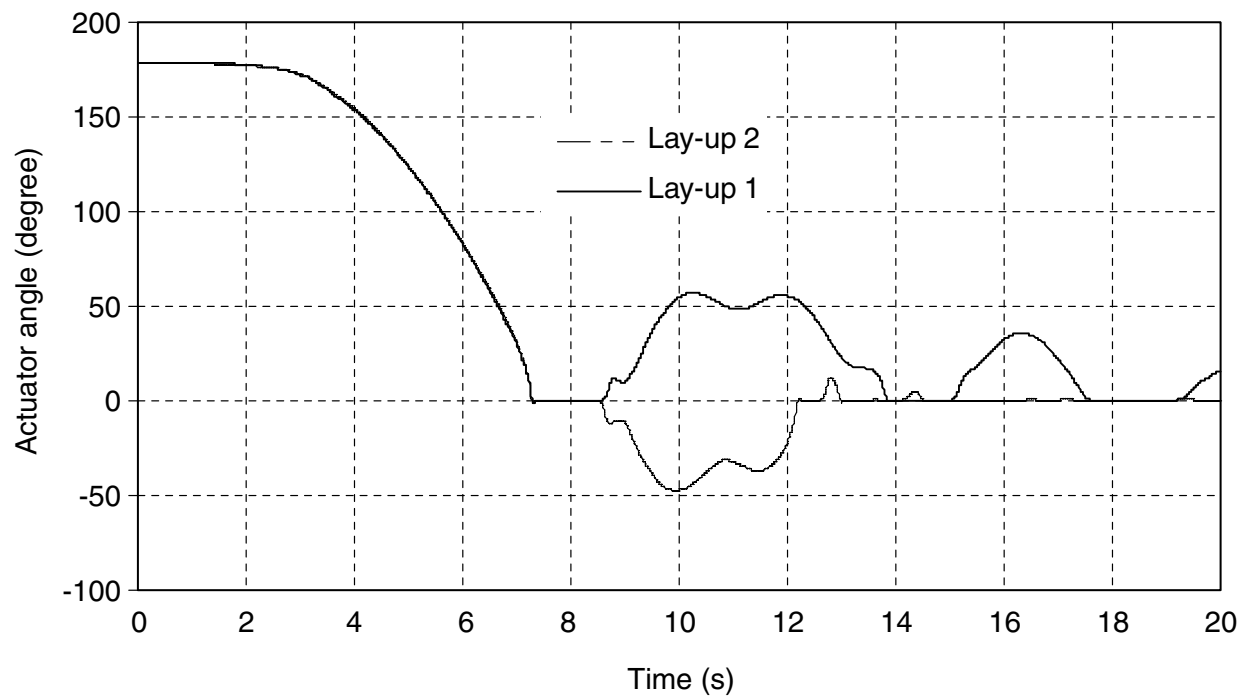

Fig. 13. Actuator angle for the flexible antenna in the first phase with different lay-ups of composite materials. 

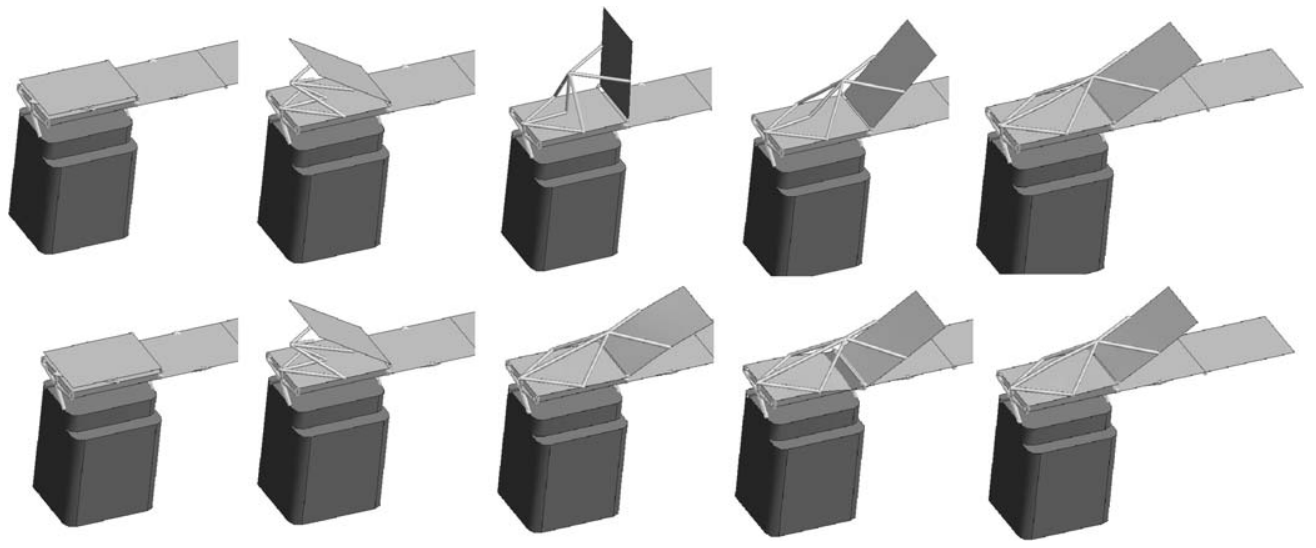

Fig. 14. First phase of the unfolding process of the antenna: rigid model (above) and flexible model (below).

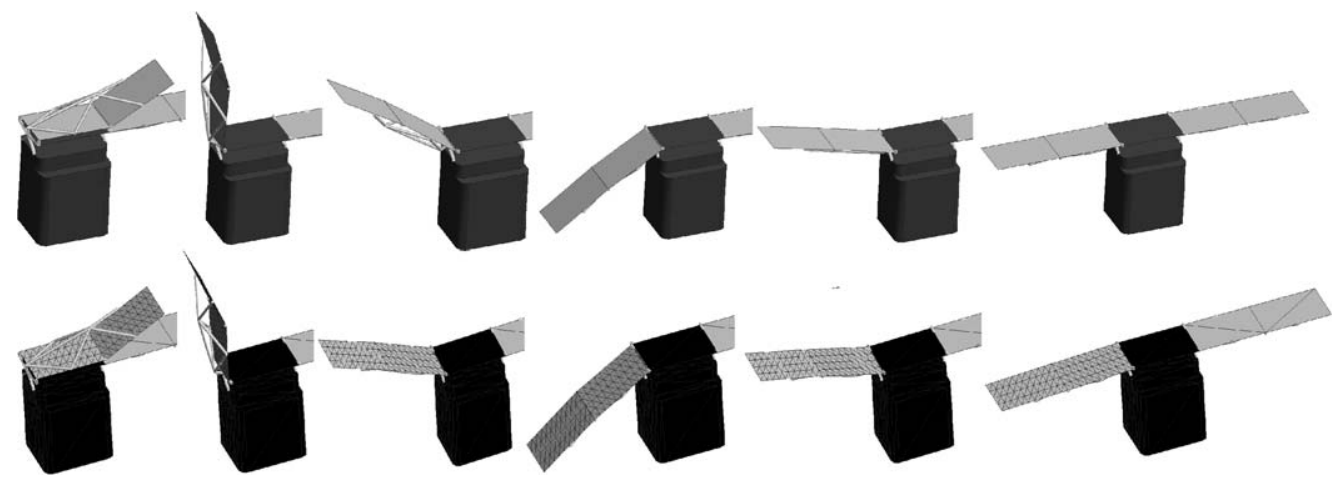

Fig. 15. Second phase of the unfolding process: rigid model (above) and flexible model (below).

\section{Conclusions}

A multibody based methodology for the analysis of flexible systems made of composite materials has been proposed in this work. The formulation used a finite element based description of the flexibility of each body referred to a body fixed coordinate system. Within the local body fixed coordinate system the deformations of the bodies were considered to be small. Furthermore, the material models used in the applications of the methodology presented are limited to be linear elastic. With these assumptions mode component synthesis can be used for flexible multibody formulation in order to reduce the number of equilibrium equations used to solve the problem.

The application of the methodology was demonstrated through numerical examples consisting of a synthetic aperture radar (SAR) antenna, which is an extremely flexible mechanism. First, it was shown that the use of rigid body models can hide some of the potential problems that exist in this type of systems by preventing the prediction of different types of motion. The flexible multibody model of the antenna uncovered a new configuration for the start of the unfolding motion and enabled corrective measures to be taken. The use of composite materials in the model of the antenna allowed the evaluation of the importance of different lay-ups sequences in the system response emphasizing the drawbacks of the oversimplification of the models made of isotropic materials, which, in the case of the SAR antenna unfolding, leads to the need of using a smaller moment for the actuator that started the mechanism.

\section{Acknowledgments}

The authors gratefully acknowledge the data provided by Prof. Andrés Kecskemethy and Dr. Martin Anantharaman for the satellite antenna deployment and Profs. Hodges and Olivier Bauchau that provided the code VABS that was of major importance in the development of this work.

\section{References}

[1] J.O. Song, E.J. Haug, Dynamic analysis of planar flexible mechanisms, Comput. Methods Appl. Mech. Engrg. 24 (1980) $359-381$. 


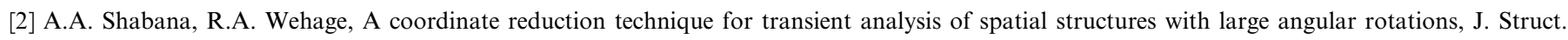
Mech. 11 (1989) 401-431.

[3] J.T. Spanos, W.S. Tsuha, Selection of component modes for flexible multibody simulation, J. Guid. Control Dyn. 14 (1991) $278-286$.

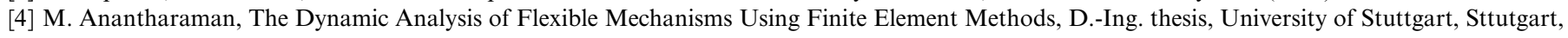
Germany, 1987.

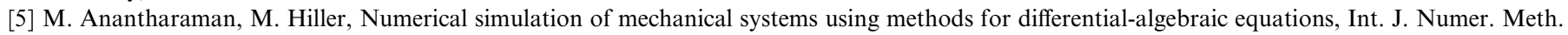
Engrg. 32 (1991) 1531-1542.

[6] F. Melzer, Symbolic computations in flexible multibody systems, Nonlinear Dyn. 9 (1996) $147-163$.

[7] J.P. Meijaard, Validation of flexible beam elements in dynamics programs, Nonlinear Dyn. 9 (1996) $21-36$.

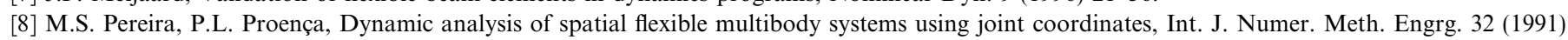
1799-1812.

[9] T. Kane, R. Ryan, A. Banerjee, Dynamics of a cantilever beam attached to a moving base, AIAA J Guid. Control Dyn. 10 (1987) 139-151.

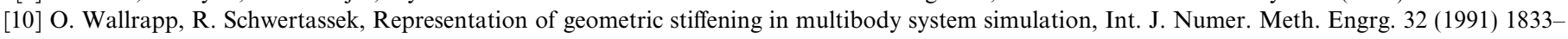
1850 .

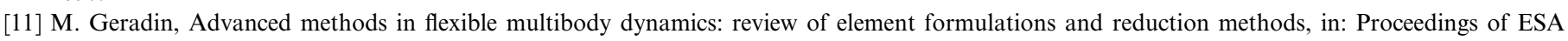
International Workshop on Advanced Mathematical Methods in the Dynamics of Flexible Bodies, ESA, Noordwijk, The Netherlands, June 3-5, 1996, pp. 83-106.

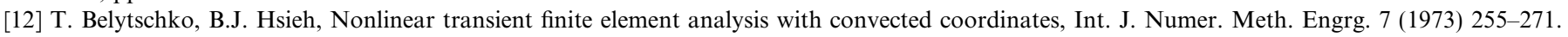

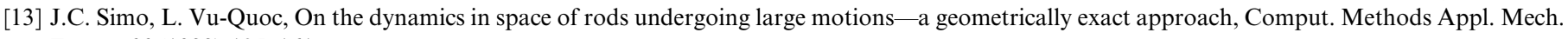
Engrg. 66 (1988) 125-161.

[14] K.-J. Bathe, S. Bolourchi, Large displacement analysis of three-dimensional beam structures, Int. J. Numer. Methods Engrg. 14 (1979) 961-986.

[15] A. Cardona, M. Geradin, A beam finite element non linear theory with finite rotations, Int. J. Numer. Methods Engrg. 26 (1988) $2403-2438$.

[16] M. Geradin, A. Cardona, A modelling of superelements in mechanism analysis, Int. J. Numer. Methods Engrg. 32 (1991) $1565-1594$.

[17] A. Shabana, Definition of the slopes and the finite element absolute nodal coordinate formulation, Multibody Syst. Dyn. 1 (1997) 339-348.

[18] J. Ambrósio, P. Nikravesh, Elastic-plastic deformations in multibody dynamics, Nonlinear Dyn. 3 (1992) 85-104.

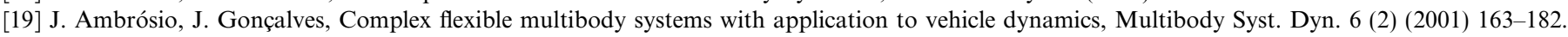

[20] O.A. Bauchau, D.H. Hodges, Analysis of nonlinear multibody systems with elastic couplings, Multibody Syst. Dyn. 3 (1999) $163-188$.

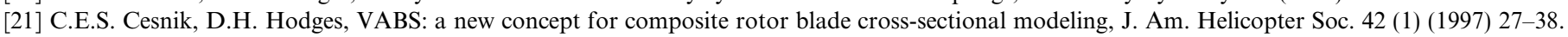

[22] I.S. Duff, A.M. Erisman, J.K. Reid, Direct Methods for Sparse Matrices, Clarendon Press, Oxford, Massachussetts, 1986.

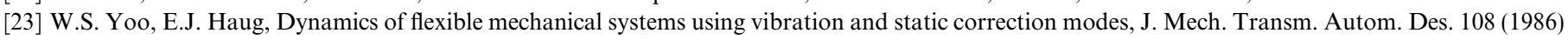
315-322.

[24] R.K. Cavin, A.R. Dusto, Hamilton's principle: finite element method and flexible body dynamics, AIAA J. 15 (12) (1977) 1684-1690.

[25] D.H. Hodges, A review of composite rotor blade modeling, AIAA J. 28 (3) (1990) 561-565.

[26] J.N. Reddy, Mechanics of Laminated Composite Plates: Theory and Analysis, CRC Press, Boca Raton, Florida, 1997.

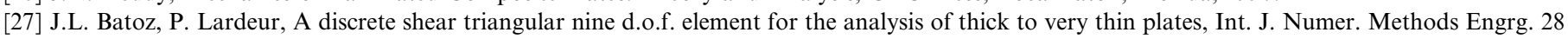
(1989) 533-560.

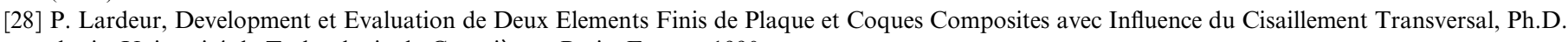
thesis, Université de Technologie de Compiègne, Paris, France, 1990.

[29] O.O. Ochoa, J.N. Reddy, Finite Element Analysis of Composite Laminates, Kluwer Academic Publishers, Dordrecht, The Netherlands, 1992.

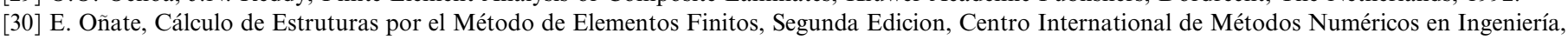
Barcelona, Spain, 1995.

[31] R.D. Cook, Concepts and Applications of Finite Element Analysis, second ed., Wiley, New York, NY, 1987.

[32] W. Yu, D.H. Hodges, On Timoshenko-like modeling of initially curved and twisted composite beams, Int. J. Solids Struct. 39 (2002) 5101-5121.

[33] B. Popescu, D.H. Hodges, On asymptotically correct Timoshenko-like anisotropic beam theory, Int. J. Solids Struct. 37 (2000) 535-558.

[34] P.E. Nikravesh, Computer-Aided Analysis of Mechanical Systems, Prentice-Hall, Englewood-Cliffs, New Jersey, 1988.

[35] H.G. Schaeffer, MSC/NASTRAN Primer-Static and Normal Modes Analysis, Schaeffer Analysis Inc., 1982. 